Research Article

\title{
Dynamic Responses of the Metro Train's Bogie Frames: Field Tests and Data Analysis
}

\author{
Xuhui He $\mathbb{D}^{1,2,3}$ Kehui Yu, ${ }^{1}$ Chenzhi Cai $\mathbb{D}^{1,2}$ and Yunfeng Zou $\mathbb{D}^{1,2}$ \\ ${ }^{1}$ School of Civil Engineering, Central South University, Changsha, Hunan, China \\ ${ }^{2}$ National Engineering Laboratory for High Speed Railway Construction, Changsha, China \\ ${ }^{3}$ Joint International Research Laboratory of Key Technology for Rail Traffic Safety, Changsha, China
}

Correspondence should be addressed to Chenzhi Cai; chenzhi.cai@csu.edu.cn

Received 28 October 2019; Revised 24 December 2019; Accepted 4 January 2020; Published 23 January 2020

Academic Editor: Mohammad Rafiee

Copyright (C) 2020 Xuhui He et al. This is an open access article distributed under the Creative Commons Attribution License, which permits unrestricted use, distribution, and reproduction in any medium, provided the original work is properly cited.

This paper focuses on the dynamic characteristics of the metro train's bogie frames based on the field test data. The acceleration signals of both motor bogie frame and trailer bogie frame of a standard B-type metro train were measured. Running tests on the Metro line 21 of Guangzhou (China) were carried out. The acquired acceleration signals of bogie frames were analyzed through several methods to identify the dynamic characteristics of the motor and trailer bogies in the time-frequency domain. The spectral analysis and time-frequency representations show that noise components exist in the high-frequency domain of the original signal, especially for the acceleration signal of the motor bogie frame. Then, the soft thresholding process and discrete wavelet transform decomposition process are conducted to obtain a denoised version of the original signals in the time-frequency domain. The vibration frequency domain and energy distribution of bogie frames under different train speeds are analyzed. The track irregularity wavelength of the metro line is calculated and analyzed based on the measured bogie frames' acceleration signals. The dynamic characteristics of the metro train's bogie frames in this paper can be adopted as a reference in the track diagnosis of the elevated metro line.

\section{Introduction}

According to recent statistics, the number of operating metro lines achieves to 185 with a total length of $5761.4 \mathrm{~km}$ among 35 cities in China at the end of 2018. The main types of metro include the underground metro and elevated metro. Compared with the underground metro, the elevated metro takes the advantages of short construction period, low cost, and energy saving in operation. Therefore, the elevated metro has been widely adopted in recent years in China to expand the metro network system, especially in highly populated cities as Beijing, Shanghai, and Guangzhou. However, the dynamic impact of the moving trains on the infrastructures concerning elevated metro and underground metro is rather different. The strong vibrations of the bridge structure of the elevated metro not only directly influence the working state and serviceability of the bridge, but also reduce the moving stability and safety of the vehicles and deteriorate the passenger riding comfort. It is therefore that many researchers and engineers around the world have devoted their attention to the investigation of the vehiclebridge interaction theoretically and experimentally. A lot of achievements have been made and are documented in numerous pieces of literature [1-4].

In addition to concerns of the dynamic response of bridges in preliminary investigations for sake of the structural safety, the condition of the railway track is rather significant for the safety and comfort of the metro operation system. The dynamic responses of the metro train are significantly sensitive to the track irregularity quality. The classification of the wavelength of irregularity is illustrated in Table 1 referring to Esveld [5]. There are several standards, for instance, EN 13848-1, EN 13848-5, EN 14363, and EN 15610, have been published in European for track maintenance. The track irregularities of high-speed railway were measured over the past five years in China. The typical wavelengths of high-speed railway are $1.4 \mathrm{~m}, 3.3 \mathrm{~m}, 6.5 \mathrm{~m}$, and $20 \mathrm{~m} \mathrm{[6].} \mathrm{The} \mathrm{track} \mathrm{irregularity} \mathrm{quality} \mathrm{also} \mathrm{has}$ 
TABLE 1: Classification of track irregularity.

\begin{tabular}{lc}
\hline Wavelength $(\mathrm{m})$ & Type of track irregularity \\
\hline $0.03-0.1$ & Short wavelength corrugation \\
$0.1-1.0$ & Medium wavelength corrugation \\
$1-3$ & Long waves and rolling defects \\
$3-25$ & Alignment, cant, twist, gauge, etc \\
$25-70$ & Alignment \\
$>70$ & Design geometry \\
\hline
\end{tabular}

influences on the dynamic behavior of maglev train. The maglev stator plane irregularities are better than normal railway rail irregularities for the wavelength of track irregularity in the range of $5-100 \mathrm{~m}$ and are inferior in the range of 1-5 $\mathrm{m}$ [7]. Zhou et al. [8] analyzed the relationship of running speed and track irregularity wavelength numerically. Their results showed that the track wavelengths are $1.4 \mathrm{~m}$ and $2.6 \mathrm{~m}$ corresponding to train speeds of $30 \mathrm{~km} / \mathrm{h}$ and $73 \mathrm{~km} / \mathrm{h}$, respectively. Some researchers had investigated the relationship of wavelengths of track irregularity and environmental vibration and noise. The wavelength of track irregularity in the range of $0.1-1 \mathrm{~m}$ has significant effects on the railway ground-borne noise [9]. Field tests and numerical models are used for studying the specific faults, such as dipped joint, raised joints, welds, crossings, and short rail head defects in literature studies [10-14].

However, it is very difficult to build an accurate mathematical model for track defect diagnosis due to the complex dynamic train system. Nowadays, some special trains and test instruments have been applied to the track diagnosis [15-17]. However, such systems are very expensive and time-consuming. Besides, these systems can only be operated at midnight when the daily service of the metro is stopped. It is therefore that several noncontact systems combined with standard train are developed for continuous measurement and diagnosis of track defects in the past several decades [18-21]. The response of the moving trains offers abundant information for other applications, for instance, riding comfort evaluation, safety, and health state assessment of the vehicle [22]. As the core component of the train, the bogie supports the car body and provides the acceptable ride levels of the running performance. At the same time, it controls the wheelset to satisfy the conflicting requirements of running stability on straight-line track and good curving performance on curved track. The dynamic response of bogie frames has a significant influence on the stability and safety of metro vehicles. Generally, the acquired test signals are nonlinear and nonstationary signals and are often mixed with a great deal of noise components due to the influence of complex vehicle structure and multiple vibration excitation sources. The recorded signal data should be processed and analyzed with the appropriate method for the sake of the most information possible [21, 23].

However, there are few investigations regarding the dynamic responses of the metro trains' bogie frames when the metro train is running on the elevated line. The aim of this paper is to investigate the dynamic characteristics of the motor and trailer bogies by adopting several signal processing methods to analyze the acquired signals from the field measurement. The acceleration signals of both motor bogie frame and trailer bogie frame of a standard B-type metro train were measured. Running tests on the Metro line 21 of Guangzhou (China) were carried out. The acquired signals are nonstationary signals and are often mixed with a great deal of noise due to the influence of complex vehicle structure and multiple vibration excitation sources. The wavelet theory has been proven its efficacy in solving nonstationary problems and advantages over alternative signal transformations. This paper uses the concept of wavelet theory, as well as several typical methods, to identify the dynamic characteristics of the motor and trailer bogies in the timefrequency domain. In order to obtain the track irregularity wavelength by an indirect way, several signal processing methods are applied to the measured bogie frames' acceleration signals. The dynamic characteristics of the metro train's bogie frames in this paper can be a reference for the evaluation of the track irregularity quality in future or other metro lines.

\section{Experimental Setup}

The standard B-type metro train was used in the experiment, which consists of 4 motor cars and 2 trailer cars, as illustrated in Figure 1. The head and trail are trailers, and 4 motor cars were arranged between the two trailer cars. The maximum speed of the B-type metro train is designed to be $120 \mathrm{~km} / \mathrm{h}$. In the running tests, sandbags with a total weight of 120 tons were distributed evenly in the test train in order to consider passengers' loads in commuter operations. The head trailer car and its adjacent motor car were chosen in the field test. Two vertical uniaxial accelerometers (type: DH1A111E, $1 \mathrm{mV} / \mathrm{g}$ sensitivity, $50 \mathrm{~g}$ full scale) were mounted on the motor bogie frame (left front side) and trailer bogie frame (left front side), respectively. The dynamic response data were collected by a 24-bit intelligent acquisition and signal processing system (type: DH5922N). The sampling frequency was chosen as $2000 \mathrm{~Hz}$ in the experiment.

Running tests were conducted between Shantian station and Zhucun station of Metro line 21 of Guangzhou during the nighttime. The test train was controlled to pass through a continuous rigid frame bridge with four spans of equal lengths of 40 meters under three different speed conditions. For constant speed cases, the test train was traveling up the bridge in respect of two different constant speeds, respectively $(80 \mathrm{~km} / \mathrm{h}$ and $115 \mathrm{~km} / \mathrm{h})$. For the braking case, the train started the brake just before the traveling of the continuous rigid frame bridge. The initial speed of the train was $70 \mathrm{~km} / \mathrm{h}$ before braking, and the whole train was intended to stop on the bridge. It should be noted that the length of a B-type metro vehicle is $19 \mathrm{~m}$, and the length of the whole train (about $120 \mathrm{~m}$ ) is less than the length of the bridge $(160 \mathrm{~m})$.

\section{Signal Processing Method}

This section briefly presents several typical signal processing methods to obtain the characteristics of the dynamic responses of bogie frames, including the fast Fourier transform, short-time Fourier transform, and wavelet transform. 


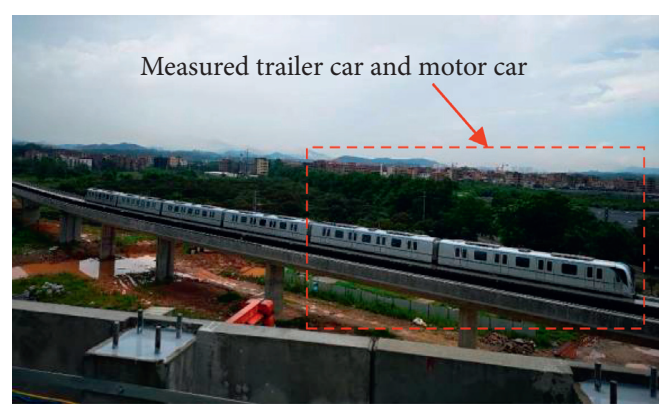

(a)

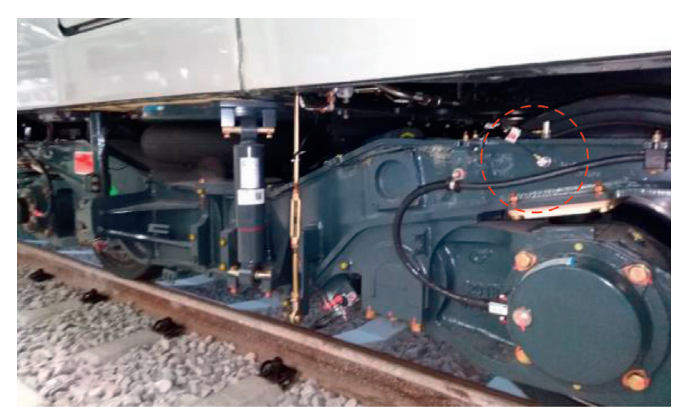

(b)

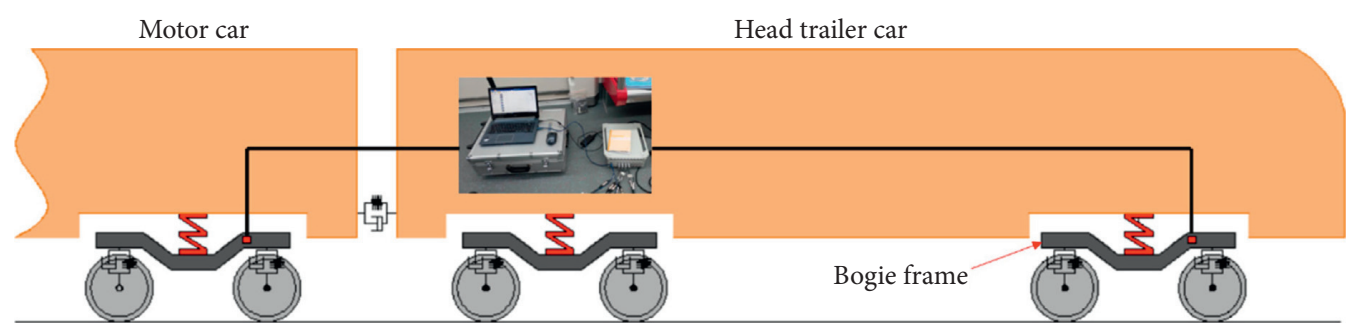

(c)

FIgURE 1: Field test setup. (a) B-type train in field test; (b) measurement points at bogie frames; (c) a schematic of the whole test system.

3.1. The Fast Fourier Transform. The fast Fourier transform (FFT) is one of the most important numerical algorithms and has been widely used in signal analysis. The original signals can be transformed from time domain to frequency domain by adopting the FFT. Then, the spectral characteristics of the signals can be extracted. The FFT could be expressed as [20]

$$
X(k)=\sum_{n=0}^{N-1} x(n) W_{N}^{k n}
$$

where $N$ represents the order number of each harmonic component, $x(n)$ represents the generic harmonic component as a complex numbers, $W_{N}=e^{-j(2 \pi k / N)}$, and $N=$ length $[x(n)]$. However, the time-varying spectral characteristics of the measured signals cannot be obtained by applying the FFT in total time domain.

3.2. The Short-Time Fourier Transform. The short-time Fourier transform (STFT) is also a Fourier-related transform used for the time-frequency analysis of a nonstationary signal. In practice, the procedure for computing STFT is to divide a longer time signal into short segments and then compute the Fourier transform separately on each shorter segment. Therefore, the plot of the time-frequency amplitude can be obtained by plotting the spectra of each segment versus time. Therefore, the STFT can overcome the time restriction in the FFT. A signal $x(t)$ is assumed to be stationary when seen through a window function $\omega(t)$, and then the STFT of the signal can be expressed as [24]

$$
X_{\tau}(f)=\int_{-T / 2}^{T / 2} x(t) \omega(t-\tau) e^{-j 2 \pi f t} \mathrm{~d} t
$$

where $T$ is the duration time of the window function $\omega(t)$ and $\tau$ represents the center time location of the window function $\omega(t)$. The STFT analysis transforms the time domain signal to a joint time-frequency distribution. However, there is a tradeoff between the time and frequency resolutions due to the assumption of the stationary of the signals within the length of the window. Better localization of the signal characteristics in time requires shorter window length. Nevertheless, the shorter window length will result in a poor frequency resolution.

3.3. Wavelet Transform. The wavelet transform is expanded on the basis of Fourier analysis to identify the time-frequency characteristics in signal processing. The wavelet transform has been widely applied as an excellent timefrequency analysis tool to analyze nonstationary signals in lots of research aspects over the last decades. Basis functions (mother wavelet) are applied in the wavelet transform to achieve the time localization of the spectral components. There are two types of wavelet transform: the continuous wavelet transform (CWT) and the discrete wavelet transform (DWT).

3.3.1. Continuous Wavelet Transform. The continuous wavelet transform of a function $f(t)$ could be defined as [18]

$$
w_{f}(a, b)=\frac{1}{\sqrt{a}} \int_{-\infty}^{\infty} f(t) \psi\left(\frac{t-b}{a}\right) \mathrm{d} t, \quad a \in R^{+} \& b \in R,
$$

where $\psi(t)$ is the mother wavelet with the scale factor $a$ and shift factor $b$ along the time domain. It can be seen from the definition that the CWT is the sum over all time of the function multiplied by scale factor and shift factor. The wavelet transform can describe the signal in time-frequency domain expression. The window in the CWT is shifted along 
the signal, and the spectrum is calculated for every position. Therefore, the CWT overcomes the disadvantage of the unalterable window size in the STFT. However, the CWT requires a considerable amount of computational work and generates a large amount of data. It is almost impractical to adopt the CWT in the analysis of long duration signals.

3.3.2. Discrete Wavelet Transform. In order to eliminate the redundant components in the CWT due to the continuous variation of the scale factor and the shift factor, the DWT adopts the discretization of the scale factor $a$ and the shift factor $b$ in terms of a power series. Specifically, the DWT adopts both the high-pass and low-pass filters to decompose the original signal into a low-frequency part $a_{j}$ (known as the "approximation") and a high-frequency part $d_{j}$ (known as the "detail"). Therefore, the decomposition of the original signal using the DWT can be described as [25]

$$
S_{j}(t)=a_{j+1}(t)+d_{j+1}(t) .
$$

where $S(t)$ is the original signal, $a(t)$ represents the approximation component, $d(t)$ represents the detail component, and the subscript $j$ represents the number of the decomposition levels. The further decomposition process of the approximate part can lead to the detail component and approximate component on a bigger scale, as illustrated in Figure 2.

According to the setting up of the sampling device, the sampling frequency of acceleration is $2000 \mathrm{~Hz}$. The original signals are resampled to $500 \mathrm{~Hz}$, and the analysis frequency is set to be $250 \mathrm{~Hz}$ in the DWT analysis. The decomposition scale is 8 , and the detailed information of these decomposition levels is demonstrated in Table 2. The "approximation" and "detail" functions of eight decomposition levels of the resampled signal of the motor bogie frame under the train speed of $115 \mathrm{~km} / \mathrm{h}$ are demonstrated in Figure 3.

In order to reveal the relationship between the vibration energy and the frequency, the total energy of acceleration signals can be calculated through the details $d(j)$. The resampled and denoised signal $S_{0}(t)$ can be decomposed into different frequency components. Thus, the vibration energy can be obtained in the form of details as follows [25]:

$$
E=\Delta t \sum_{t=0}^{t} S_{0}^{2}(t)=\sum_{j=1}^{n} \sum_{t=0}^{t} d_{j}^{2}(t)
$$

where $\Delta t$ is the time step of the acceleration signals and $n$ is the decomposition level.

3.3.3. Wavelet Denoising Process. The noise signal is usually existing in the high-frequency parts. Therefore, the reduction of the noise components in the detail is necessary. Generally, the process of the wavelet-based denoising can be described into three steps: (1) the choosing of the wavelet and the determination of the decomposition level number; (2) an appropriate threshold is selected to process the detail coefficients at each level; and (3) reconstruction of the signal through the processed wavelet coefficients. The universal "VisuShrink" threshold could be given as [26]

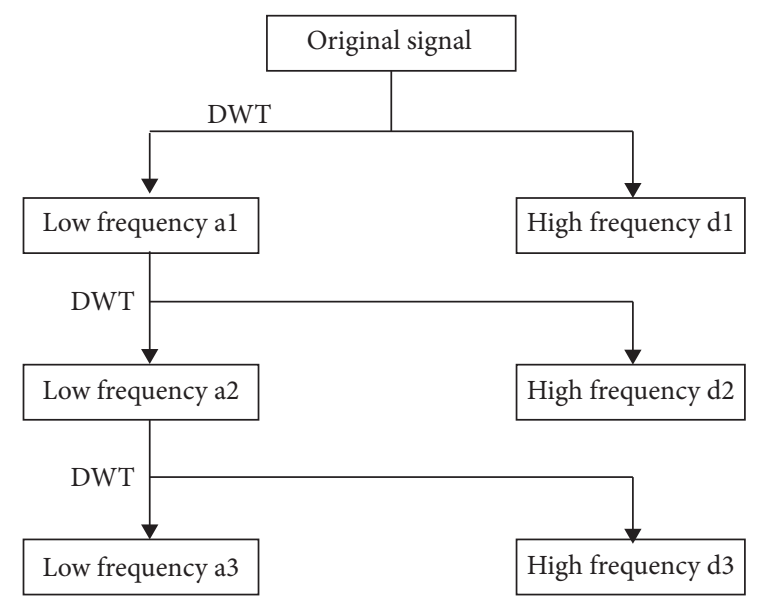

FIgURE 2: Schematic diagram of the DWT decomposition process.

$$
\text { Thr }=\delta \sqrt{2 \log (N)}
$$

where $\delta$ is the noise standard variance and $N$ is the size or length of the signal. Generally, there are two traditional algorithms of thresholding, namely, hard thresholding $\left(T_{\text {hard }}\right)$ and soft thresholding $\left(T_{\text {soff }}\right)$, and they are defined as follows:

$$
\begin{aligned}
T_{\text {soft }} & =\operatorname{sgn}(x)(|x| \cdot \mathrm{Thr}), \\
T_{\text {hard }} & =x \cdot(|x|>\text { Thr }),
\end{aligned}
$$

where $x$ is the wavelet coefficient.

In the present paper, the original acceleration signals of bogie frames will be decomposed by a db 10 wavelet (Daubechies 10) [25]. By using proposed methods to analyze the dynamic characteristics of the bogie frames based on their acceleration signals, the analysis process is schematically illustrated in Figure 4. The FFT and STFT methods will be applied to the measured acceleration data for spectral analysis and time-frequency analysis, respectively. Then, the acquired signals with a sampling frequency of $2000 \mathrm{~Hz}$ will be resampled to $500 \mathrm{~Hz}$. After the implementation of the soft thresholding process, DWT decomposition process and energy calculation will be conducted to obtain the energy distribution at each decomposition level. Based on the resampled and denoised acceleration signals, the CWT, will be introduced to obtain a denoised version of the original signals in the time-frequency domain.

\section{Results and Discussion}

4.1. FFT Analysis. The original acceleration signals of the motor bogie frame and trailer bogie frame under the train speed of $115 \mathrm{~km} / \mathrm{h}$ are demonstrated in Figure 5. The duration time of the measurement is 2 seconds when the train is running on the continuous rigid frame bridge. It can be seen from Figure 5 that the maximum amplitude of the acceleration of the motor bogie frame and trailer bogie frame is $3.58 \mathrm{~g}$ and $1.91 \mathrm{~g}$, respectively $\left(g=9.81 \mathrm{~m} / \mathrm{s}^{2}\right)$. Figure 6 illustrates the transformation of the original signals from time domain to frequency domain by using the FFT. There are two obvious frequency ingredients in the frequency 
Table 2: Frequency of each decomposition level.

\begin{tabular}{lccc}
\hline Decomposition level & Frequency $(\mathrm{Hz})$ & Decomposition level & Frequency $(\mathrm{Hz})$ \\
\hline 1 & $125-250$ & 5 & $7.81-15.625$ \\
2 & $62.5-125$ & 6 & $3.91-7.81$ \\
3 & $31.25-62.5$ & 7 & $1.96-3.91$ \\
4 & $15.625-31.25$ & 8 & $0.98-1.96$ \\
\hline
\end{tabular}
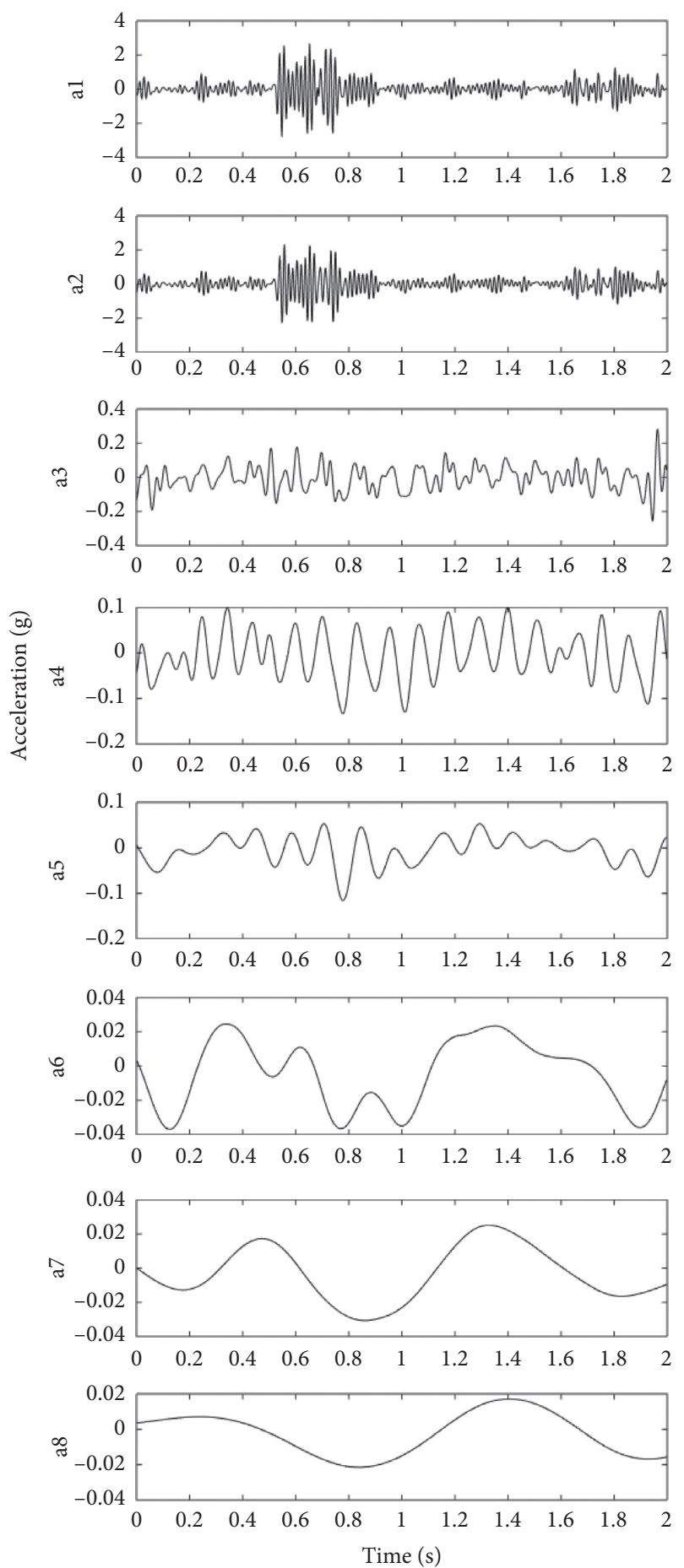

(a)
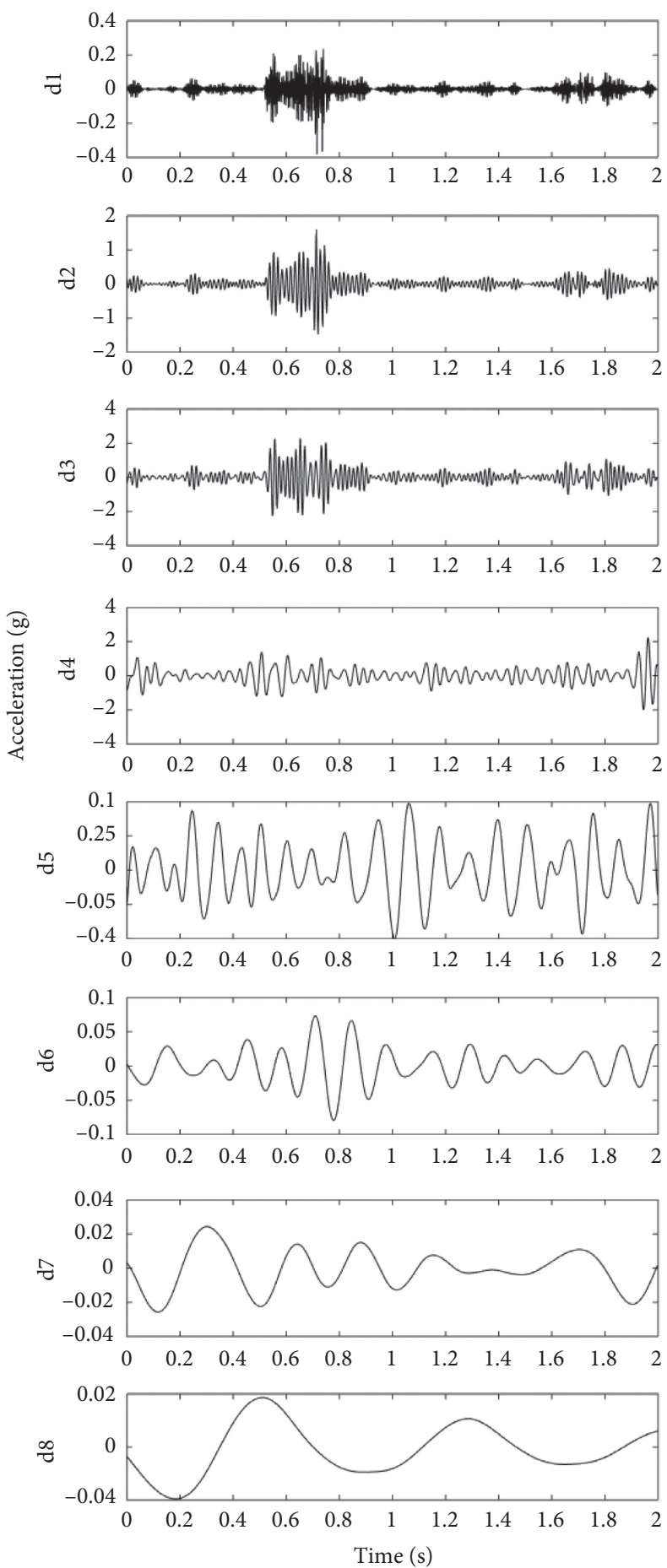

(b)

FIGURE 3: (a) Approximation and (b) detail functions of eight decomposition levels of motor bogie frame acceleration signals with a speed of $115 \mathrm{~km} / \mathrm{h}$. 


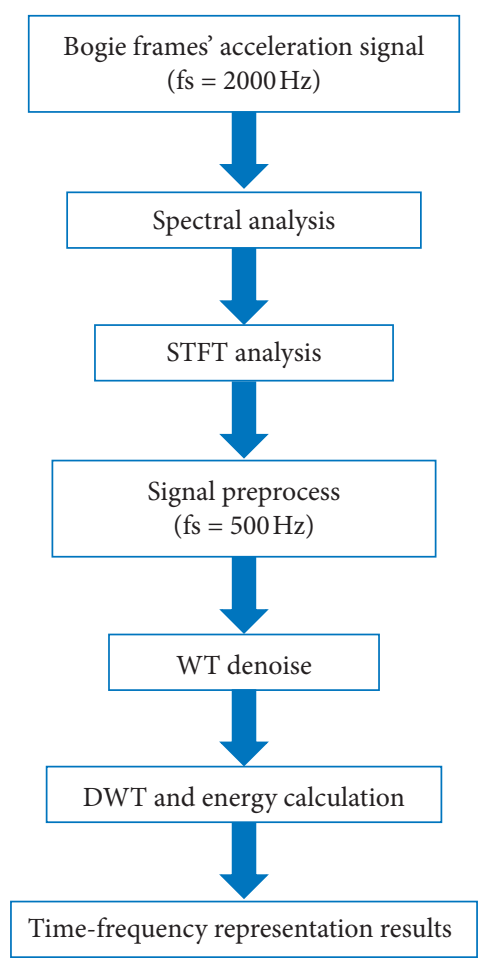

FIgURE 4: Time-frequency analysis process of the test signal.

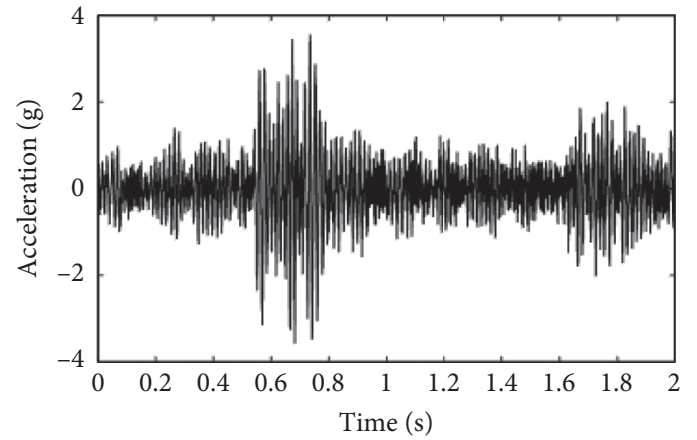

(a)

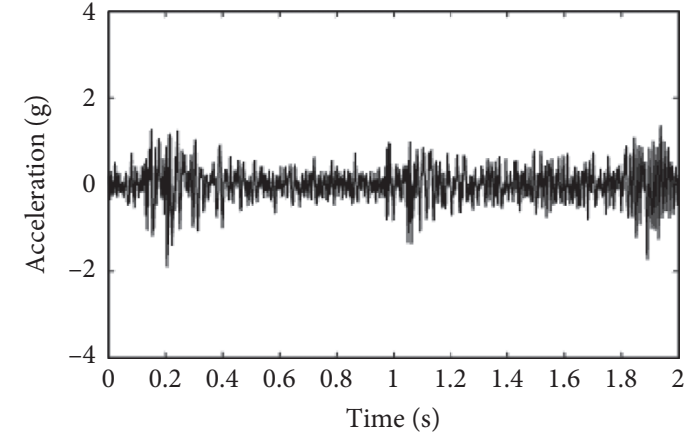

(b)

FIgURE 5: The original acceleration signals of bogie frames under the train speed of $115 \mathrm{~km} / \mathrm{h}$. (a) The motor bogie frame and (b) The trailer bogie frame.

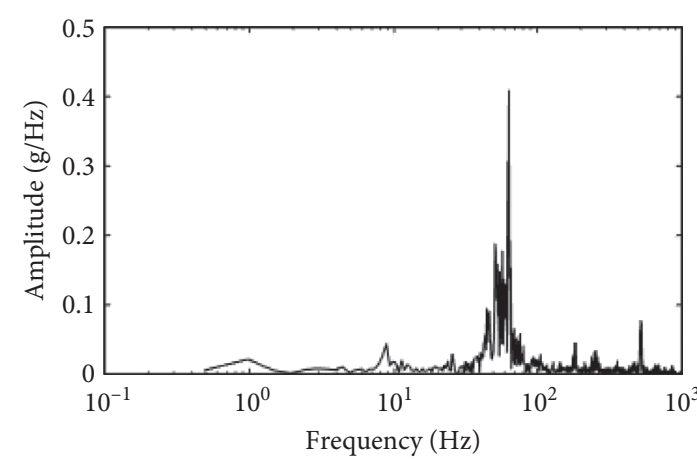

(a)

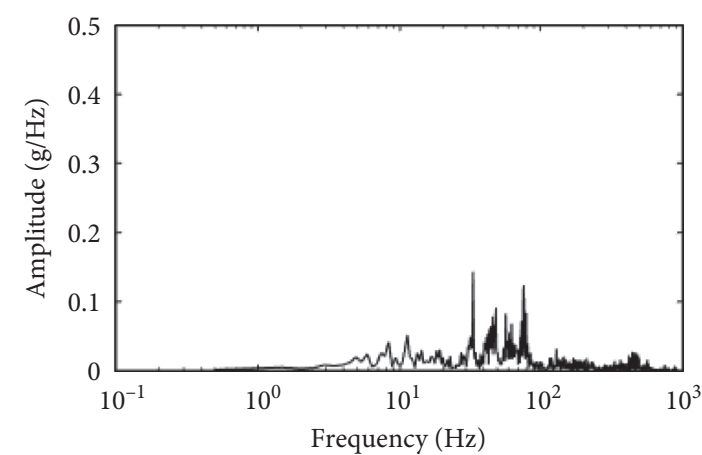

(b)

Figure 6: FFT results of the test signals. (a) The motor bogie frame and (b) The trailer bogie frame. 


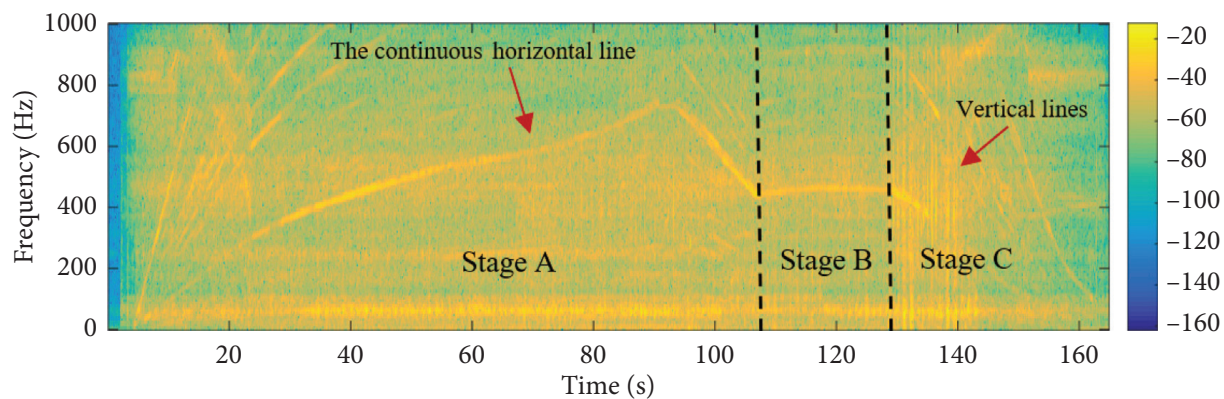

(a)

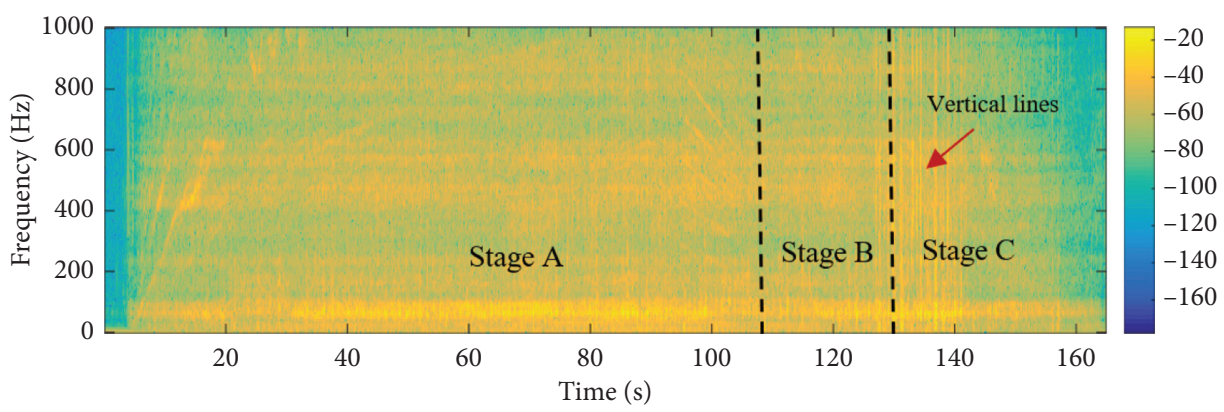

(b)

FIGURE 7: The spectrogram of bogie frames' full signals under the constant speed case of $115 \mathrm{~km} / \mathrm{h}$ : (a) motor bogie frame and (b) trailer bogie frame.

domain of the motor bogie frame's acceleration signal located at $8.8 \mathrm{~Hz}$ and $45-64 \mathrm{~Hz}$, respectively. It can be observed from Figure 6 that there are some frequency ingredients at $8.3 \mathrm{~Hz}, 11.2 \mathrm{~Hz}, 33.7 \mathrm{~Hz}$, and $45-76 \mathrm{~Hz}$ in the FFT of the trailer bogie frame's acceleration signals. Actually, satisfactory results cannot be obtained due to the timevarying characteristics of the bogie frame's acceleration signals. Therefore, the STFT method is used in the next section for the sake of the time-frequency analysis.

4.2. STFT Analysis. As aforementioned, the accelerometers have been installed at the motor bogie frame and trailer bogie frame, respectively. The STFT method is used to analyze the frequency characteristics of the bogie frame acceleration signal in time domain. The Hamming window with a length of $0.1 \mathrm{~s}$ and an overlap of $95 \%$ have been chosen in the STFT analysis [13]. Figure 7 shows the spectrogram for the motor bogie frame and trailer bogie frame. The duration time of the running test of the metro train is 145 seconds. It can be divided into three stages as preparation stage (stage A: from $0 \mathrm{~s}$ to $107 \mathrm{~s}$ ), constant stage (stage B: from $107 \mathrm{~s}$ to $128 \mathrm{~s}$ ), and braking stage (stage C: from $128 \mathrm{~s}$ to $145 \mathrm{~s}$ ). In the preparation stage, the train speed increases from 0 to $115 \mathrm{~km} /$ $h$. Then, the train runs at a constant speed of $115 \mathrm{~km} / \mathrm{h}$ from $107 \mathrm{~s}$ to $128 \mathrm{~s}$. It should be noted that the train is controlled to pass through the continuous rigid frame bridge at a constant speed of $115 \mathrm{~km} / \mathrm{h}$. Finally, the train starts the brake at the time $128 \mathrm{~s}$ and stops at the time $145 \mathrm{~s}$. A continuous horizontal line at different frequencies can be observed in Figure 7(a). It is increasing to $90 \mathrm{sec}$, and then it decreases from 90 to $105 \mathrm{sec}$, and then it remains constant. However, the continuous horizontal line cannot be observed in the spectrogram of the trailer bogie frame's signal, as illustrated in Figure 7(b). The reason for the differences may be the component of the meshing frequency of the transmission in the motor bogie. There are several vertical lines in both Figures 7 (a) and 7(b) in the range of $128 \mathrm{~s}-145 \mathrm{~s}$, which may be because of the interaction between the train and the bridge.

4.3. Energy Calculation. The nonlinear noise component in the original signals can be smoothed through threshold wavelet denoising. The energy of each detail component can be adopted to evaluate the vibration characteristics of the bogie frames. The signals of the bogie frames under three different circumstances are analyzed here. For constant speed cases, the test train was traveling up the bridge with constant speeds of $80 \mathrm{~km} / \mathrm{h}$ (Case 1) and $115 \mathrm{~km} / \mathrm{h}$ (Case 2), respectively. The braking case is also chosen and it is nominated as Case 3. For Case 3, the train started the brake just before the traveling of the continuous rigid frame bridge. The initial speed of the train was $70 \mathrm{~km} / \mathrm{h}$ before braking and the whole train was intended to stop on the bridge. The energy of details at each decomposition level and its percentage of the total energy with respect to different bogie frames in these three cases are demonstrated in Tables 3 and 4, respectively. For the motor bogie frame, the energy of third decomposition level (frequency range is $31.25 \mathrm{~Hz}-62.5 \mathrm{~Hz}$ ) has the highest energy in these three cases, and its percentage of the total energy is $53.12 \%, 53.32 \%$, and $32.29 \%$ corresponding to Case 1, Case 2, and Case 3, respectively. By the comparison of Case 1 and Case 2 presented in Table 3, we can realize that the increase of the train's speed results in a rapid growth of the energy of details at 
TABLE 3: Energy of the motor bogie frame at each decomposition level and its percentage of the total energy in respect of different cases.

\begin{tabular}{lcccccccccc}
\hline \multirow{2}{*}{ Test case } & Brand & 1 & 2 & 3 & 4 & 5 & 6 & 7 & 8 & 6 \\
& Frequency range (Hz) & $125-250$ & $62.5-125$ & $31.25-62.5$ & $15.63-31.25$ & $7.81-15.63$ & $3.91-7.81$ & $1.96-3.91$ & $0.98-1.96$ \\
\hline \multirow{2}{*}{ Case 1} & Energy $\left(\mathrm{m}^{2} / \mathrm{s}^{3}\right)$ & 0.3 & 1.36 & 3.36 & 0.49 & 0.36 & 0.24 & 0.07 & 0.14 \\
& Percentage (\%) & 4.81 & 21.44 & 53.12 & 7.71 & 5.72 & 3.84 & 1.15 & 2.21 \\
\hline \multirow{2}{*}{ Case 2 } & Energy $\left(\mathrm{m}^{2} / \mathrm{s}^{3}\right)$ & 1.59 & 7.81 & 15.61 & 1.49 & 1.32 & 0.78 & 0.37 & 0.3 \\
& Percentage $(\%)$ & 5.43 & 26.70 & 53.32 & 5.09 & 4.49 & 2.68 & 1.26 & 1.03 \\
\hline \multirow{2}{*}{ Case 3 } & Energy $\left(\mathrm{m}^{2} / \mathrm{s}^{3}\right)$ & 0.24 & 1.01 & 2.42 & 1.14 & 0.78 & 1.24 & 0.23 & 0.39 \\
& Percentage (\%) & 3.2 & 13.59 & 32.39 & 15.33 & 10.46 & 16.69 & 3.08 & 5.26 \\
\hline
\end{tabular}

TABLE 4: Energy of the trailer bogie frame at each decomposition level and its percentage of the total energy in respect of different cases.

\begin{tabular}{lcccccccccc}
\hline \multirow{2}{*}{ Test case } & Brand & 1 & 2 & 3 & 4 & 5 & 6 & 7 & 8 & 6 \\
& Frequency range $(\mathrm{Hz})$ & $125-250$ & $62.5-125$ & $31.25-62.5$ & $15.63-31.25$ & $7.81-15.63$ & $3.91-7.81$ & $1.96-3.91$ & $0.98-1.96$ \\
\hline \multirow{2}{*}{ Case 1 } & Energy $\left(\mathrm{m}^{2} / \mathrm{s}^{3}\right)$ & 0.43 & 1.83 & 4.25 & 1.52 & 1.26 & 0.68 & 0.36 & 0.18 \\
& Percentage $(\%)$ & 4.07 & 17.4 & 40.45 & 14.46 & 12.02 & 6.43 & 3.41 & 1.76 \\
\hline \multirow{2}{*}{ Case 2 } & Energy $\left(\mathrm{m}^{2} / \mathrm{s}^{3}\right)$ & 0.94 & 3.25 & 6.52 & 2.86 & 1.82 & 1.27 & 0.32 & 0.16 \\
& Percentage $(\%)$ & 5.46 & 18.96 & 38.04 & 16.67 & 10.61 & 7.44 & 1.86 & 0.95 \\
\hline \multirow{2}{*}{ Case 3 } & Energy $\left(\mathrm{m}^{2} / \mathrm{s}^{3}\right)$ & 0.76 & 3.03 & 3.82 & 1.59 & 0.98 & 0.56 & 0.35 & 0.08 \\
& Percentage $(\%)$ & 6.8 & 27.14 & 34.17 & 14.27 & 8.83 & 5.02 & 3.09 & 0.68 \\
\hline
\end{tabular}
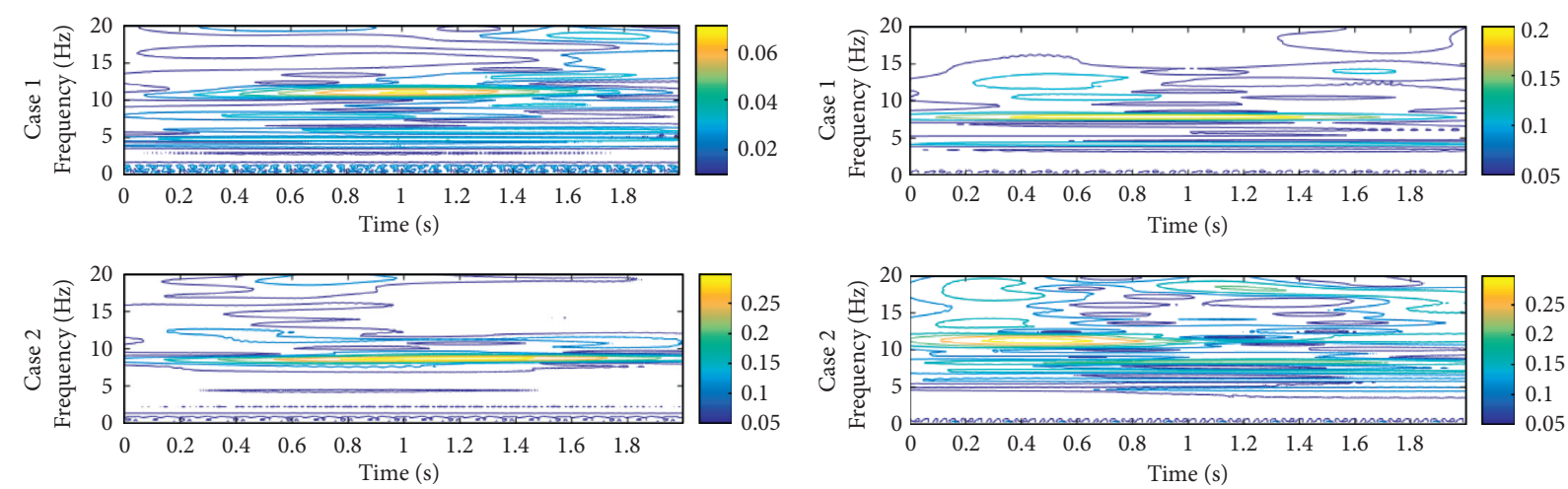

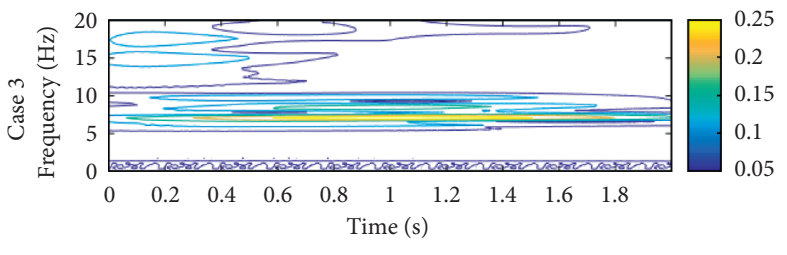

(a)

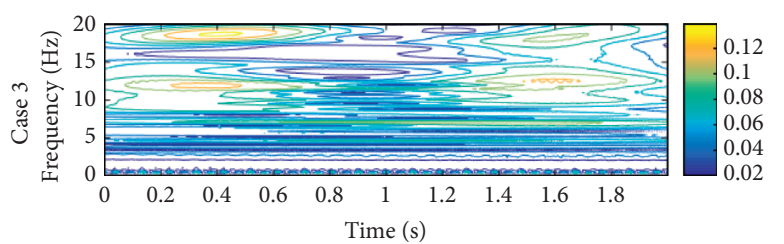

(b)

FIgURE 8: Time-frequency representation of motor and trailer bogie frames' acceleration signals in respect of different cases: (a) motor bogie frame and (b) trailer bogie frame.

each decomposition level. However, its percentage of the total energy does not change much. The trailer bogie frame under Case 1 and Case 2 has similar phenomenon, as demonstrated in Table 4. It should be noted that there is about $5.26 \%$ of total energy in the motor bogie frame at the frequency domain of $0.98-1.96 \mathrm{~Hz}$ under the braking case (Case 3). Such distribution of energy is different from the energy distribution of the motor bogie frame and trailer bogie frame in other cases.

4.4. Characteristic Frequency. The acceleration signals of bogie frames with respect to the above three cases (Case 1, Case 2, and Case 3) are used to investigate the dynamic behavior of bogie frames, as are the characteristics of track irregularity. The wavelength of track irregularity can be calculated according to the formula $\lambda=v / f$ (where $v$ represents the running speed of the train and $f$ is the dominant frequency of bogie frame's acceleration signals) [12]. Generally, the railway track irregularities appear in the form of short waves $(3-25 \mathrm{~m})$. The speed of the test train in this paper varies from $70 \mathrm{~km} / \mathrm{h}$ to $115 \mathrm{~km} / \mathrm{h}$. It is therefore that frequency components above $20 \mathrm{~Hz}$ in the decomposed signal have been removed.

The aforementioned vertical signals of Case 1, Case 2, and Case 3 are used to investigate the wavelength of track irregularity. The CWT method with a Morlet basis has been 
TABLE 5: Characteristic frequency.

\begin{tabular}{|c|c|c|c|c|c|}
\hline Case & Bogie frame & Frequency $1(\mathrm{~Hz})$ & Frequency $2(\mathrm{~Hz})$ & Wavelength $1(\mathrm{~m})$ & Wavelength $2(\mathrm{~m})$ \\
\hline \multirow{2}{*}{ Case 1} & Motor & 11.23 & - & 1.98 & - \\
\hline & Trailer & 7.81 & 一 & 2.85 & - \\
\hline \multirow{2}{*}{ Case 2} & Motor & 8.79 & - & 3.63 & - \\
\hline & Trailer & 8.3 & 11.23 & 3.85 & 2.84 \\
\hline \multirow{2}{*}{ Case 3} & Motor & 7.32 & - & 2.65 & - \\
\hline & Trailer & 11.72 & 18.55 & 1.65 & 1.04 \\
\hline
\end{tabular}

applied to achieve the time-frequency representations of bogie frames' acceleration signals [18]. Then, the dominant frequency of the bogie frame's acceleration signals can be obtained. Figure 8 demonstrates time-frequency representations of motor and trailer bogie frames' acceleration signals in respect of different cases. It can be observed from Figure 8 that the dominant frequencies of Case 1 are around $11.23 \mathrm{~Hz}$ and $7.81 \mathrm{~Hz}$ corresponding to motor bogie frame and trailer bogie frame, respectively, and dominant frequencies of Case 2 are $8.79 \mathrm{~Hz}$ and $8.3 \mathrm{~Hz}$ corresponding to motor bogie frame and trailer bogie frame, respectively. The dominant frequency of bogie frames is not sensitive to the speed of the train. For Case 3 (the braking case), there exist two dominant frequencies $(11.72 \mathrm{~Hz}$ and $18.55 \mathrm{~Hz})$ in the trailer bogie frame's acceleration signals. However, there is still only one dominant frequency around $7 \mathrm{~Hz}$ in the motor bogie's acceleration signals of Case 3 . Once the dominant frequency has been obtained, the wavelength of track irregularity can be determined, as illustrated in Table 5. The minimum wavelength of track irregularity is $1.04 \mathrm{~m}$ in the braking case of the trail bogie frame's acceleration signal. The maximum wavelength of track irregularity is $3.85 \mathrm{~m}$ in Case 2 of the trail bogie frame's acceleration signal. The wavelengths of track irregularity in the narrow band of $0.9-2.2 \mathrm{~m}$ and $2.2-3.5 \mathrm{~m}$ are mainly caused by the periodic composition of the rail during the rolling process [14]. The present study can be adopted as a reference in the track diagnosis of the elevated metro line.

\section{Conclusions}

This paper focuses on the dynamic characteristics of the metro train's bogie frames based on the field test data. Several timefrequency analysis methods are used to distinguish the dynamic characteristics of both the motor bogie frame and the trailer bogie frame. The denoising, decomposition, and reconstruction processing are conducted according to the wavelet theory. The energy distributions of the motor bogie frame and trailer bogie frame under different cases are calculated based on different decomposition levels. The energy of the third decomposition level with a frequency range of $31.25 \mathrm{~Hz}-62.5 \mathrm{~Hz}$ has the highest energy in both cases with respect to both bogie frames. For constant speed cases, the increase of the train's speed results in a rapid growth of the energy of details at each decomposition level. However, its percentage of the total energy does not change much. There is about $5.26 \%$ of total energy in the motor bogie frame at the frequency domain of $0.98-1.96 \mathrm{~Hz}$ under the braking case, and its energy distribution is different from the constant speed cases. The acceleration signals of bogie frames have been used to investigate the track irregularity by using the wavelet transform method. The results show that the track irregularity wavelengths of the investigated viaduct metro line are about 1-4 $\mathrm{m}$. The present study can be adopted as a reference in the track diagnosis of the elevated metro line.

\section{Data Availability}

The data of this study are available from the corresponding author upon request.

\section{Conflicts of Interest}

The authors declare that there are no conflicts of interest regarding the publication of this paper.

\section{Acknowledgments}

The authors gratefully acknowledge the support of the National Natural Science Foundations of China (Grant nos. U1534206, 51925808, and U1934209), the National Key R\&D Program of China (Grant no. 2017YFB1201204), and Guangzhou Metro Group.

\section{References}

[1] M. X. D. Li, E. G. Berggren, M. Berg, and I. Persson, "Assessing track geometry quality based on wavelength spectra and track-vehicle dynamic interaction," Vehicle System Dynamics, vol. 46, no. sup1, pp. 261-276, 2008.

[2] I. I.-Y. Choi, J.-H. Um, J. S. Lee, and H.-H. Choi, "The influence of track irregularities on the running behavior of highspeed trains," Proceedings of the Institution of Mechanical Engineers, Part F: Journal of Rail and Rapid Transit, vol. 227, no. 1, pp. 94-102, 2012.

[3] A. Haigermoser, B. Luber, J. Rauh, and G. Gräfe, "Road and track irregularities: measurement, assessment and simulation," Vehicle System Dynamics, vol. 53, no. 7, pp. 878-957, 2015.

[4] X. He, Y. Gai, and T. Wu, "Simulation of train-bridge interaction under wind loads: a rigid-flexible coupling approach," International Journal of Rail Transportation, vol. 6, no. 3, pp. 163-182, 2018.

[5] M. Podwórna, "Modelling of random vertical irregularities of railway tracks," International Journal of Applied Mechanics and Engineering, vol. 20, no. 3, pp. 647-655, 2015.

[6] X. Bian, H. Jiang, C. Chang, J. Hu, and Y. Chen, "Track and ground vibrations generated by high-speed train running on ballastless railway with excitation of vertical track irregularities," Soil Dynamics and Earthquake Engineering, vol. 76, pp. 29-43, 2015. 
[7] J. Shi, W.-s. Fang, Y.-j. Wang, and Y. Zhao, "Measurements and analysis of track irregularities on high speed maglev lines," Journal of Zhejiang University SCIENCE A, vol. 15, no. 6, pp. 385-394, 2014.

[8] D. Zhou, P. Yu, L. Wang, and J. Li, “An adaptive vibration control method to suppress the vibration of the maglev train caused by track irregularities," Journal of Sound and Vibration, vol. 408, pp. 331-350, 2017.

[9] S. L. Grassie, "Rail irregularities, corrugation and acoustic roughness: characteristics, significance and effects of reprofiling," Proceedings of the Institution of Mechanical Engineers, Part F: Journal of Rail and Rapid Transit, vol. 226, no. 5, pp. 542-557, 2012.

[10] X. Yang, S. Gu, S. Zhou, J. Yang, Y. Zhou, and S. Lian, "Effect of track irregularity on the dynamic response of a slab track under a high-speed train based on the composite track element method," Applied Acoustics, vol. 99, pp. 72-84, 2015.

[11] X. Zhao, Z. Li, and R. Dollevoet, "The vertical and the longitudinal dynamic responses of the vehicle-track system to squat-type short wavelength irregularity," Vehicle System Dynamics, vol. 51, no. 12, pp. 1918-1937, 2013.

[12] S. Bogdan, "Interrelation between wavelengths of track geometry irregularities and rail vehicle dynamic properties," Archives of Transport, vol. 25-26, pp. 97-108, 2013.

[13] P. Salvador, V. Naranjo, R. Insa, and P. Teixeira, “Axlebox accelerations: their acquisition and time-frequency characterisation for railway track monitoring purposes," Measurement, vol. 82, pp. 301-312, 2016.

[14] Y. He and Z. Li, "Characteristic analysis of track spectrums of different subway line conditions," Journal of Railway Engineering Society, vol. 8, pp. 99-104, 2014.

[15] International Union of Railways, UIC Code 518, Testing and Approval of Railway Vehicles from the Point of View of Their Dynamic Behaviour-Safety-Track Fatigue-Ride Quality, International Union of Railways, Paris, France, 2003.

[16] Ministry of Railways of the People's Republic of China, TI310761-2013 High-Speed Railway Engineering Dynamic Acceptance Technical Criterion, Ministry of Railways of the People's Republic of China, Beijing, China, 2013.

[17] Federal Railroad Administration, VTI, Vehicle/Track Interaction Safety Standards; High-Speed and High Cant Deficiency Operations, Federal Railroad Administration, Washington, DC, USA, 2013.

[18] A. Caprioli, A. Cigada, and D. Raveglia, "Rail inspection in track maintenance: a benchmark between the wavelet approach and the more conventional Fourier analysis," $\mathrm{Me}$ chanical Systems and Signal Processing, vol. 21, no. 2, pp. 631-652, 2007.

[19] L. Chiacchiari and G. Loprencipe, "Measurement methods and analysis tools for rail irregularities: a case study for urban tram track," Journal of Modern Transportation, vol. 23, no. 2, pp. 137-147, 2015.

[20] J. Ning, J. Lin, and B. Zhang, "Time-frequency processing of track irregularities in high-speed train," Mechanical Systems and Signal Processing, vol. 66-67, pp. 339-348, 2016.

[21] J. S. Lee, S. Choi, S.-S. Kim, C. Park, and Y. G. Kim, “A mixed filtering approach for track condition monitoring using accelerometers on the axle box and bogie," IEEE Transactions on Instrumentation and Measurement, vol. 61, no. 3, pp. 749-758, 2012.

[22] I. Simon, Handbook of Railway Vehicle Dynamics, CRC Press, Boca Raton, FL, USA, 1st edition, 2006.

[23] H. C. Tsai, C. Y. Wang, and N. E. Huang, "Fast inspection and identification techniques for track irregularities based on
HHT analysis," Advances in Adaptive Data Analysis, vol. 4, pp. 1-26, 2012.

[24] Y. Zhang, Z. Guo, W. Wang, S. He, T. Lee, and M. Loew, “A comparison of the wavelet and short-time Fourier transforms for Doppler spectral analysis," Medical Engineering \& Physics, vol. 25, no. 7, pp. 547-557, 2003.

[25] M. A. Sayed, M. R. Kaloop, E. Kim, and D. Kim, "Assessment of acceleration responses of a railway bridge using wavelet analysis," KSCE Journal of Civil Engineering, vol. 21, no. 5, pp. 1844-1853, 2017.

[26] S. A. Chouakri, F. B. Reguig, S. Ahmaldi, and O. Fokapu, "Wavelet denoising of the electrocardiogram signal based on the corrupted noise estimation," Computers in Cardiology, vol. 32, pp. 1021-1024, 2005. 